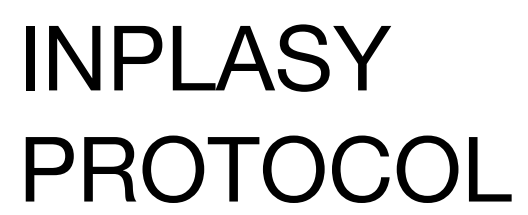

To cite: Moreno - Sepulveda et al. Natural cycles in frozenthawed embryo transfer are associated with lower risks of preeclampsia and large-forgestational-age infants than artificial cycles: A systematic review and meta-analysis. Inplasy protocol 202060088. doi:

10.37766/inplasy2020.6.0088

Received: 23 June 2020

Published: 23 June 2020

Corresponding author: Jose Moreno - Sepulveda

jmorenos@gmail.com

Author Affiliation:

Universitat Autonoma de Barcelona

Support: None.

Review Stage at time of this submission: Data analysis.

Conflicts of interest: None to declare.

\section{Natural cycles in frozen-thawed embryo transfer are associated with lower risks of preeclampsia and large-for-gestational-age infants than artificial cycles: A systematic review and meta-analysis}

Moreno - Sepulveda1', J; Espinos², J; Checa, M³.

Review question / Objective: Is a natural cycle (NC-FET) safer than an artificial cycle (AC-FET) for perinatal and maternal outcomes in FET cycles?

Condition being studied: The freeze-all strategy has reported successful results with higher live birth rates and lower incidence of ovarian hyperstimulation syndrome compared to fresh embryo transfer (ET). Singletons born after FET also have better neonatal outcomes compared with singletons born after fresh ET regarding low birth weight (LBW), small for gestational age (SGA), and preterm birth (PTB). However, compared with fresh ET, FET seems to carry a greater risk of hypertensive disorders in pregnancy (including gestational hypertension and preeclampsia), being born as large for gestational age (LGA) and macrosomia. The reasons behind these findings are not clearly understood, but it was suggested that some cryoprotectants or the vitrification and thawing process per se could develop some metabolic or epigenetic changes related to abnormal placentation and eventually preeclampsia. Most of FET cycles are carried out after endometrial preparation through the administration of exogenous hormones. However, the altered levels of estradiol and progesterone that could be reached during the artificial cycle frozen embryo transfer (AC-FET) could favour some endometrial changes that may trigger an impaired decidualization and placentation, leading to the development of placenta-related complications such as hypertensive disorders of pregnancy.

INPLASY registration number: This protocol was registered with the International Platform of Registered Systematic Review and Meta-Analysis Protocols (INPLASY) on 23 June 2020 and was last updated on 23 June 2020 (registration number INPLASY202060088).

\section{INTRODUCTION}

Review question / Objective: Is a natural cycle (NC-FET) safer than an artificial cycle
(AC-FET) for perinatal and maternal outcomes in FET cycles? 
Rationale: It is hypothesized that the hormonal endometrium preparation used for the thawed embryo transfer may contribute to the development of hypertensive disorders in pregnancy, preeclampsia, large for gestational age, and macrosomia. We conducted a systematic review and meta-analysis to assess if natural cycle frozen embryo transfer is safer than artificial cycle frozen embryo transfer for perinatal and maternal outcomes.

Condition being studied: The freeze-all strategy has reported successful results with higher live birth rates and lower incidence of ovarian hyperstimulation syndrome compared to fresh embryo transfer (ET). Singletons born after FET also have better neonatal outcomes compared with singletons born after fresh ET regarding low birth weight (LBW), small for gestational age (SGA), and preterm birth (PTB). However, compared with fresh ET, FET seems to carry a greater risk of hypertensive disorders in pregnancy (including gestational hypertension and preeclampsia), being born as large for gestational age (LGA) and macrosomia. The reasons behind these findings are not clearly understood, but it was suggested that some cryoprotectants or the vitrification and thawing process per se could develop some metabolic or epigenetic changes related to abnormal placentation and eventually preeclampsia. Most of FET cycles are carried out after endometrial preparation through the administration of exogenous hormones. However, the altered levels of estradiol and progesterone that could be reached during the artificial cycle frozen embryo transfer (AC-FET) could favour some endometrial changes that may trigger an impaired decidualization and placentation, leading to the development of placenta-related complications such as hypertensive disorders of pregnancy.

\section{METHODS}

Search strategy: An electronic search was performed in the following databases: PubMed/MEDLINE, SCOPUS and the
Cochrane database, from 1982 through March 2020. We also searched the reference lists of relevant articles for any additional studies not covered by the literature search. The search combined terms and descriptors related to variants for the interventions, population study and outcomes: IVF with or without intracytoplasmic sperm injection (ICSI), frozen-thawed embryo transfer, endometrial preparation, natural cycle, hormone replacement cycle, hypertensive disorders in pregnancy, preeclampsia, large for gestational age, macrosomia, preterm birth, post-term birth, low birth weight, very low birth weight, small for gestational age, stillbirth, neonatal mortality, gestational diabetes, cesarean section, placenta previa, placenta accreta, placental abruption and postpartum haemorrhage. The search strategy was modified to fit with the syntaxes used in each database consulted.

Participant or population: Frozen embryo transfers in IVF/ ICSI patients.

Intervention: Natural cycle frozen embryo transfer (NC-FET).

Comparator: Artificial cycle frozen embryo transfer (AC-FET).

Study designs to be included: Observational studies.

Eligibility criteria: We included only studies that compared the reproductive outcomes between NC-FET and AC-FET in IVF/ICSI cycles.

Information sources: PubMed/MEDLINE, SCOPUS and the Cochrane database, from 1982 through April 2020.

Main outcome(s): The primary outcome measure was the rate of adverse pregnancy outcomes including: Hypertensive disorders in pregnancy (HDP), defined as a blood pressure of $\geq 140 / 90$ $\mathrm{mmHg}$ on two or more occasions, at least 6 hours apart, and more than 20 weeks of gestation; gestational hypertension, defined as hypertension arising de novo 
after 20 weeks' gestation in the absence of proteinuria and without biochemical or haematological abnormalities; preeclampsia, diagnosed by the presence of de novo hypertension after 20 weeks' gestation accompanied by proteinuria and/ or evidence of maternal acute kidney injury, liver dysfunction, neurological features, hemolysis or thrombocytopenia, and/or fetal growth restriction; large for gestational age (LGA), defined as birth weight above the 90th percentile; and macrosomia defined as birth weight above $4000 \mathrm{~g}$.

Additional outcome(s): Secondary outcomes measures were preterm birth (PTB) defined as a live birth before 37 weeks; post-term birth defined as a live birth after 42 weeks; low birth weight (LBW) defined as a birth weight below $2500 \mathrm{~g}$; very low birth weight (VLBW) defined as a birth weight below $1500 \mathrm{~g}$; small for gestational age (SGA), defined in two ways: Birth weight under 2 standard deviations or below the 10th percentile; stillbirth defined as the death of a fetus prior to the complete expulsion or extraction from its mother after 22 completed weeks of gestational age; neonatal mortality defined as death before 28 days postpartum; gestational diabetes, cesarean section, placenta previa, placenta accreta, placental abruption and postpartum haemorrhage.

Data management: In a first screening, two independent authors (J.M, M.C) assessed all of the abstracts retrieved from the search, and then they obtained the full manuscripts of citations that fit the inclusion criteria. They judged study eligibility, assessed quality, and extracted data solving discrepancies by agreement, and if needed, reaching a consensus with a third author (J.E). Next, the full texts of eligible articles were obtained to evaluate the eligibility of the studies that fit the inclusion criteria. Data extracted from all studies was summarized for each outcome listed below. The authors referred to the Grading of Recommendations Assessment, Development and Evaluation (GRADE) to evaluate the quality of evidence for each outcome.
Quality assessment / Risk of bias analysis: We followed the guidelines suggested by the Newcastle-Ottawa Scales (NOS) for assessing the quality of included studies. The studies were evaluated on the selection process, comparability of cohorts and outcomes ascertainment.

Strategy of data synthesis: To determine the pooled effect of each variable, we used a Mantel-Haenszel model and applied the fixed-effects model. The risk ratio (RR) for dichotomous data accompanied by the $95 \%$ confidence intervals (Cls) were calculated. Statistical significance was set at a P-value $50 \%$, we applied the randomeffects model (21). We used the Review Manager (RevMan Version 5.3 Software, Copenhagen, Denmark) for statistical analysis.

\section{Subgroup analysis: None.}

Sensibility analysis: Sensitivity analyses were performed to examine the influence of variation among studies on the overall risk estimates.

Language: English, spanish.

Country(ies) involved: Spain, Chile.

Keywords: frozen-thawed embryo transfer; natural cycle; artificial cycle; hypertensive disorders of pregnancy; preeclampsia; macrosomia; perinatal.

Dissemination plans: Our aim is to publish this study in a high impact scientific journal

\section{Contributions of each author:}

Author 1 - Jose Moreno-Sepulveda - J.M designed the study and collected the data. Also he analysed the data and drafted the first manuscript. All authors interpreted the pooled data, critically revised the manuscript for important intellectual content and approved the final version.

Author 2 - Juan Jose Espinos - J.E analysed the data and drafted the first manuscript. All authors interpreted the pooled data, critically revised the manuscript for important intellectual content and approved the final version. 
Author 3 - Miguel Angel Checa - M.C. designed the study and collected the data. All authors interpreted the pooled data, critically revised the manuscript for important intellectual content and approved the final version. 